\title{
Erratum
}

\section{Explaining synchrony in feed-forward networks:}

\section{Are McCulloch-Pitts neurons good enough?}

\section{T. Nowotny, R. Huerta}

Institute for Nonlinear Science, UCSD, 9500 Gilman Drive, La Jolla, CA 92093-0402, USA

Published online: 4 December 2003

Biol Cybern 89, 237-241 (2003)

Unfortunately, the rubric for the article "Explaining synchrony in feed-forward networks: Are McCulloch-Pitts neurons good enough?" by T. Nowotny und R. Huerta was omitted. The rubric should have been Letter to the Editors. We apologize for this oversight. 